\title{
Medical staffing crisis in psychiatry
}

\author{
Rachel Jenkins and Jan Scott
}

\begin{abstract}
This article summarises the findings of a National Health Service Execultive, Royal College of Psychiatrists', National Association of Health Authorities and Trusts, and Trust Federation working group on medical staffing in mental health, which reported to ministers in summer 1996 and was launched at a conference in November 1996 jointly with the College. Although primarily focused on England and Wales, it is clear that similar problems exist in other parts of the United Kingdom and the Republic of Ireland.
\end{abstract}

\section{Origins of the stafiing crisis}

The changing pattern of mental health services delivery is very labour intensive work, and has been accompanied by the development of major staffing shortages at both consultant psychiatrist and training grade levels. In England and Wales, $10 \%$ of consultant psychiatrist posts are vacant, one in five advisory appointment committees are cancelled through lack of appropriate applicants, and one in 10 do not make an appointment because of the lack of an appropriate candidate. Furthermore one in three newly advertised consultant posts are filled by established consultants moving from one post to another rather than by a new consultant.

This crisis in medical staffing in psychiatry is of serious concern and has arisen for a number of reasons. While there has been an expansion in the number of consultant posts available, the expansion of junior posts have not kept pace, leaving a shortfall in the number of appropriately trained applicants for consultant posts. In addition the senior registrar numbers agreed by the Joint Planning Advisory Committee were not funded in full so that, at a time when it would have been possible to recruit senior registrars, not even the intended numbers of senior registrars were appointed because of the high number of unfunded posts.

Factors such as the number of demands currently made on consultant psychiatrists, the media presentation of community psychiatry and the distressing experience of psychiatrists who have been criticised in some recent enquiries into local tragedies, may also be contributing directly or indirectly to lowering of morale among consultant psychiatrists, with many established consultants moving into the private sector or taking early retirement. This has been particularly noticeable in the south of England with 35 consultants prematurely leaving the North Thames region in the past two years.

Recruitment difficulties are more serious in certain areas of the country. The College has noted that posts are difficult to fill where they are poorly resourced in terms of services for patients and where the catchment area is large, and a recent National Association of Health Authorities and Trusts (NAHAT) survey indicated that rural trusts had greater recruitment difficulties.

The present shortage of consultants is likely to get worse because the implementation of the specialist training grade may lead to a loss of junior staff to undertake call duty, thus further increasing the workload of already overloaded consultant staff. New deal monies have been used to take some of the pressure off medical staff in mental health services. However, psychiatry has already developed sophisticated multi-disciplinary working and skill-mix. Further opportunities for skill-mix and multidisciplinary working should be pursued but they are more likely to reduce the stress level within a post rather than lead to significant reductions in the required number of medical staff. There are statutory Mental Health Act responsibilities that require senior clinical staff, and clinical supervision and accountability must be maintained, both in order to train the psychiatrists of the future and for the purposes of clinical responsibility for patient care.

Urgent action is therefore required in the short-, medium- and longer-term to overcome the medical staffing problems in mental health which will otherwise destabilise the existing services.

\section{The way ahead}

\section{Short-term action}

Short-term action focuses on recruitment and retention of consultants, and managing the implementation of the specialist training grade. 
There are four main approaches: broadening the field of recruitment; enabling part-time working for those not presently in work and increased sessions for those already working part-time; encouraging recently retired consultants to return on a sessional basis; and discouraging consultants from leaving the National Health Service (NHS) prematurely by improving job plans and working conditions.

The working group considered the potential for recruitment from overseas and, while specifically not wishing to draw experienced staff from countries with their own staff shortages, it appears there is scope for recruitment from the USA where there is a surplus of qualified psychiatrists. The College can now make available an information pack containing relevant details for obtaining registration, work permits, entry permits, etc., as well as information on how to advertise in the American Psychiatric Association job bank.

Broadening the field of recruitment to the USA may make a small impact on the current difficulties, but it is more vital to ensure that the current consultant population is retained and developed. This requires support of individuals in these posts, greater awareness by purchasers and providers of the demands of this type of work and greater flexibility in how service needs are met, for example by the creation of options for part-time or job share posts, and providing greater variability in the weekly work programme. The special needs of psychiatry with regard to the instigation of the Calman recommendations should be closely examined to ensure that appropriate funding is available. Trust managers need to identify those areas where consultants can be better supported, and thereby enabled to make a more effective contribution and encouraged to remain in post, for example enabling specialisation, academic links, protected research sessions, flexibility in working hours, opportunities to learn from other services which have successfully negotiated and adopted models of service delivery, greater support of continuing professional development, including payment of registration fees and journal fees, the funding of sabbaticals, and better secretarial and administrative support. There would of course be a cost to some of this, but trusts have already demonstrated a willingness to pay the 'market rate' for consultants in other shortage specialities. The additional costs would be offset against the overheads of employing locums, and the enhanced value of a substantive appointment.

\section{Medium-term action}

In the medium term, an increase in the number of individuals eligible to apply for substantive consultant posts is required. It is important to encourage locum consultants and some of individuals in non-training career grade posts to acquire appropriate skills and qualifications to allow them to seek a consultant appointment. It is also crucial to ensure that those who wish to train flexibly in psychiatry can also be accommodated.

The National Health Service Executive (NHSE) is exploring how the appointment of flexible trainees can be accelerated. A mechanism is needed to support postgraduate deans in their efforts to ensure that flexible training is available to as many individuals as possible.

The College has identified over 1000 doctors who occupy non-training grade career posts, of whom a minority will be eligible to enter training grades and to take the membership exam. However, it is vital that career advisors ensure that junior doctors do not move prematurely from training posts into non-career posts. The College will continue to widely advertise its affiliate scheme to ensure that the maximum number of trainees undertake continuing professional development and as many as possible take the membership exam.

Little information is available about junior doctors who leave psychiatry for reasons other than failure to gain appropriate professional qualification. There is concern both about the direct impact of poor services on junior doctors working within them, as well as the indirect impact via the lowered morale of consultants who provide training. The College is planning a questionnaire survey of junior doctors who leave psychiatry to explore their reasons for doing so.

Lastly, we need to ensure that appropriate numbers of medical school graduates and junior doctors enter the training. We welcome the fact that psychiatry is retained as a core undergraduate speciality. The General Medical Council (1993) document Tomorrow's Doctors should raise the profile of psychiatry teaching in medical schools as a core speciality in the undergraduate curriculum. However, additional action is required to improve the public image of psychiatry, to reduce the stigma attached to mental illness and to providers of mental health services in the community at large, but also within medicine.

\section{Conclusions}

If we are to develop a sustainable medical staffing strategy for psychiatry it is clear that we need more information about staff flows through the system. Two studies are currently underway which will help to remedy this information deficit. The first is a study of individuals who left NHS consultant posts within the past two years, which the NHSE has funded the 
College to carry out. The second is a joint venture between the RCPsych and the NHS Women's Unit who are undertaking a questionnaire survey of the career aspirations of flexible trainees in psychiatry to see if there is a need to encourage the development of part-time consultant posts for a significant number of these individuals. The results will be made available by the NHSE to health authorities and trusts, and it is likely that they will indicate the need for greater flexibility in the career structure in psychiatry e.g. job shares, part-time consultant posts and options of rotating posts at consultant level.

If we are to increase the numbers available at consultant level in the long-term we need to increase the numbers of medical graduates recruited into psychiatry. While these numbers have not fallen (recent surveys suggest that recruitment of medical graduates into psychiatry has remained steady at $\sim 4 \%$ ), a long-term strategy to recruit more medical graduates will only happen if they perceive that consultant psychiatry posts offer an attractive, fulfilling and sustainable career option.

Improving the quality of posts requires immediate action in order to retain the staff we currently have. Therefore this report should not be viewed as a simple 'wish list', but rather as an essential template for action if we are to reverse the trends that have made psychiatry a shortage speciality.

Purchasers are a major group psychiatry needs to target if we are to achieve the above objectives. Because purchasing tends to be predicated on the annual contracting round, this has militated against a coherent local staffing strategy to improve the numbers and quality of both junior and senior posts. We need sufficient junior posts to train the consultants for the future, and we need sufficient consultant posts to train those juniors. The consultant posts also need to be sufficiently attractive to retain high-quality staff.

At the conference, the President of the College made a vital plea to the profession that despite the stress consultant staff find themselves under, we must ensure that we do not pass on our unhappiness, concern and angst to juniors who may feel overwhelmed at the prospect of their future and leave the speciality prematurely. He emphasised that we should learn the lessons from primary care where six years ago, general practitioners were so unhappy with their new contract that they passed their disenchantment to aspiring general practitioner trainees and medical students and what had been an extremely popular speciality suddenly lost much of its appeal.

Work is now well underway in all these areas, both separately and collaboratively between NHSE, the College, NAHAT and postgraduate deans, and the report has been disseminated widely. It is hoped that this energetic approach to solving the manpower crisis in psychiatry will serve as a template for action in other shortage specialities.

\section{Acknowledgements}

We wish to thank Dr Kendell, President of the Royal College of Psychiatrists, and Sheila Adams of the NHSE for helpful comments on an earlier draft of this paper.

\section{Reference}

General Medical Council (1993) Tomorrows Doctors. London: GMC.

NATIONAL HEALTH SERVICE EXECUTIVE (1996) Medical Staffing Issues in Psychiatry: Problems and Potential Solutions. London: Mental Health Division of NHSE.

Rachel Jenkins, Principal Medical Officer, WHOUK Office, Institute of Psychiatry, London SE5 $8 A F$, and *Jan Scott, Professor of Community Psychiatry, University of Newcastle upon Tyne, Newcastle upon Tyne NE1 4LP

*Correspondence 